\title{
Primary Balloon Angioplasty Combined with Balloon-Assisted Maturation of Autogenous Arteriovenous Fistula in Patients with Small-Caliber Vasculature
}

\author{
Ahmed Kamal Gabr, MD; ${ }^{1}$ Ahmed Khairy Allam, MD; ${ }^{2}$ Tarek Elsayed Abouelregal, MD ${ }^{3}$ \\ ${ }^{1}$ Department of Vascular Surgery, Ain Shams University. ${ }^{2}$ Department of General Surgery, Vascular \\ Surgery unit, Benha University. ${ }^{2}$ Department of Vascular Surgery, Nile Insurance Hospital. ${ }^{3}$ Internal Medicine \\ Department, Cairo University.
}

Background: Failure or delayed maturation of arteriovenous fistula (AVF) in patients with end-stage renal disease (ESRD) who require hemodialysis (HD) can lead to catheter-related complications as bacteremia and sepsis with the resultant high morbidity and mortality. Small- diameter veins are often a limiting factor for the successful creation of arteriovenous fistulas (AVFs). Balloon-assisted maturation (BAM) is a recent, innovative, yet controversial method for developing autogenous arterio-venous fistulae (AVF).

Purpose: Is to evaluate the use of intraoperative primary balloon angioplasty (PBA) as a technique to upgrade the small-caliber or diameter veins during AVF construction in conjunction with sequential balloonassisted maturation to salvage failing fistulas, expedited maturation \& improve the patency of AVFs.

Materials and methods: Prospectively collected data over a 2-year period. Between January 2016 and December 2017, 121 patients who underwent AVF creation in two tertiary referral hospitals in Saudi Arabia, Vascular surgery Department, Nile Insurance Hospital \& Vascular Surgery Unit, Benha University/Department of Surgery, Egypt, were retrospectively analyzed. The duration to maturation of the AVF was determined by comparing the period between the time of creation of the fistula and the first successful cannulation of the fistula for patients with ESRD on hemodialysis. Patients with peritoneal dialysis were excluded. Patients who underwent BAM or construction of AVF at an outside institution were excluded. Follow-up consisted reviewing of postoperative AVF duplex for patency, hemodialysis units, vascular and nephrology clinics databases, and telephone interviews. Successful outcome was determined as the functional ability to use the fistula for hemodialysis without surgical revision.

Results: Of the 136 PBA procedures, $119(87.5 \%)$ remained patent and subsequently underwent BAM with a resulting functional AVF. These consisted of 106 of the original AVFs and 13 new AVFs created at other sites. Occlusions occurred in 30 of the 121 fistulas (24.8\%), and 15 were salvaged using BAM techniques. The mean balloon diameter for all dilatations was $3.5 \mathrm{~mm}$. Of the initial 121 AVFs placed, 106 (87.6\%) were made functional and 30 failed due to occlusion. Fifteen were salvaged using recanalization techniques and sequential BAM. Of the remaining fifteen patients, 11 were unsalvaged and received AVFs at another site using PBA and BAM techniques and 4 required graft placement. One AVF had to be ligated secondary to steal syndrome with insertion of chronic hemodialysis catheter as an alternative access. Overall 116 patients $(95.9 \%)$ received working AVFs. All fistulas were functioning at 90 days after the final BAM. Graft placement was necessary in only 4 of the 121 patients (3.3\%).

Conclusion: The combination between PBA and BAM is an overall approach that facilitates AVF maturation, improves function, prolongs patency, and facilitates use of small-caliber veins as an autogenous vascular access. The shorter maturation times have reduced the overall need for indwelling catheters with their associated risks. The resulting large-diameter AVFs are easy to cannulate and are associated with improved flow and patency rates. Successful AVFs have been created using veins that would have otherwise been deemed unusable. Adoption of this conjoint technique should help meet the stringent demands for autogenous access placement defined by KDOQI and Fistula First Breakthrough Initiative (FFBI) and, thereby, improve the duration and quality of life for the hemodialysis patients.

Key words: Balloon angioplasty, maturation, hemodialysis, radio-cephalic arteriovenous fistula, vascular access failure.

Abbreviations: AVF (arteriovenous fistula), ESRD (end-stage renal disease), BAM (balloon-assisted maturation), HD (hemodialysis), ESRD (end-stage renal disease), PBA (Primary Balloon Angioplasty), FFBI (Fistula First Breakthrough Initiative), KDOQI (Kidney Disease Outcomes Quality Initiative). 


\section{Introduction}

The arteriovenous fistula (AVF) is the access of choice for hemodialysis $(H D)^{1}$ for both primary and secondary hemodialysis access based on their improved patency, reduced costs, and lower rate of infections relative to both catheters and prosthetic arteriovenous accesses (AVGs), but its success as an access is limited by a high rate of maturation failure (MF). ${ }^{2}$ Therefore, an upsurge of new techniques and studies has emerged in an effort to increase maturation and salvage rates in AVFs. ${ }^{3}$ The National Kidney Foundation-Kidney Disease Outcomes Quality Initiative (NKF-KDOQI) and the Society for Vascular Surgery recommend an arterio-venous fistula (AVF) should be placed at least 6 months before the anticipated need for hemodialysis (HD) ${ }^{4,5} \mathrm{NKF}-\mathrm{KDOQI}$ also recommends the creation of an AVF in $65 \%$ of patients who elect to undergo HD. These recommendations in combination with the Fistula First Breakthrough Initiative (FFBI) have since resulted in an increased number of fistulae created for HD access. ${ }^{5,6}$

Consequently, this has resulted in an increased incidence of non-maturation or delayed maturation of AVF. 4-6 The delay in maturation of AVF has previously been linked to increased health care cost, higher catheter-related sepsis with resultant morbidity due to infection, cardiac events, and all-cause mortality. 5,7,8 Thus, this emphasis on the placement of AVFs, however, has inadvertently resulted in an increase in the number of nonmaturing AVFs. ${ }^{9}, 10$ The most important factor limiting the AVF growth and patency is the availability of a venous segment with adequate diameter: cephalic veins smaller than $3 \mathrm{~mm}$ in diameter were reported to increase immediate failure rate and to decrease primary patency rate. ${ }^{11,12} \mathrm{~A}$ developing technique in angioplasty is now being used that proposes to improve maturation times. This procedure attempts to promptly mature AVF by serial graduated dilatation of the entire fistula length and is postulated to accelerate its maturation process. It is referred to as balloon-assisted maturation (BAM) for AVF. ${ }^{13-17}$ During BAM, angioplasty of the entire fistula is done repeatedly every 2 weeks with a serial increase in balloon diameters until maturation. In an effort to increase the number of primary AVFs, shortens maturation times, and reduces the number of indwelling catheters, it has been developed two conjoint techniques in this context designed to create and expedite the use of a functioning AVF. These techniques include primary balloon angioplasty (PBA) during fistula construction in selected patients with small-caliber veins, in conjunction with sequential balloonassisted maturation (BAM) of the inflow, usable segment for cannulation and, and in some patients, the outflow tract; and surveillance fistulograms (SFGs) with preemptive intervention. ${ }^{18}$ The aim of this study is to evaluate the use of intraoperative primary balloon angioplasty (PBA) as a technique to upgrade the small-caliber or diameter veins during AVF construction in conjunction with sequential balloon-assisted maturation to salvage failing fistulas, expedited maturation \& improve the patency of AVFs.

\section{Patients and methods}

We conducted a retrospective analysis of prospectively collected data over a 2-year period inbetween January 2016 and December 2017 for 121 patients who underwent PBAs during their AVF creation using an intention-to-treat all-autologous policy at 2 tertiary referral centers in Saudi Arabia (Security Forces Hospital Program \& Al-Noor Specialist Hospital-Makkah), Vascular Surgery Department, Nile Insurance Hospital \& Vascular Surgery Unit, Banha University/Department of Surgery, Egypt. The study protocol was approved by the local hospital's Ethical and Scientific Review Board in the enrolled hospitals. All the procedures were based on the cephalic vein, and there was no involvement of the basilic vein in the study.

All patients with suboptimal vein diameter $(\leq 3$ $\mathrm{mm}$ ) during the creation of AVF were ballooned up 1 to $1.5-\mathrm{mm}$ larger than the nominal vein diameter.

Accordingly, veins $<3 \mathrm{~mm}$ were excluded from our study. Table 1 . The duration required for maturation of the AVF was determined by comparing the period between the time of creation of the fistula and the first successful cannulation of the fistula for patients with ESRD on hemodialysis. Patients with peritoneal dialysis were excluded. Patients who underwent BAM or construction of AVF at an outside institution were also excluded. Follow-up consisted of reviewing the postoperative AVF duplex study for patency, hemodialysis units, vascular and nephrology clinics databases. Successful outcome was determined as the functional ability to use the fistula for hemodialysis without surgical revision. A thorough preoperative evaluation was performed in the 121 patients receiving AVF as a vascular access. Physical examination included upper arm inspection with and without tourniquet placement to determine site preference. The non-dominant upper extremity was favored for access placement whenever feasible. Preoperative vein mapping was used in all cases. ${ }^{19}$ Veins $<3.0 \mathrm{~mm}$ in diameter (measured by ultrasound with a tourniquet, outer wall-to-outer wall) were considered suboptimal and enrolled in the study. Intraoperative diameters were measured with a combination of calipers and Garrett dilators in all patients who underwent PBA before AVF creation. Measurements were confirmed by venography on the first BAM performed in all patients who had documented veins $<3 \mathrm{~mm}$ 
based on dilation with an initial 3-mm balloon to nominal pressure. PBA was performed through the spatulated end of the venotomy over an angled-tip 0.035 -inch hydrophilic wire $(0.014$-inch for $2.5-\mathrm{mm}$ balloons). Veins with diameters between 1 and 3 $\mathrm{mm}$ underwent balloon angioplasty 1 - to $1.5-\mathrm{mm}$ larger than the nominal vein diameter from the middle forearm to the spatulated end of the vein.

Spatulated veins were initially hydrostatically dilated with a 21- gauge angio-catheter before measurement. In the case of a radial-cephalic access, the site of anastomosis is frequently moved more proximal on the radial artery to allow for retro-grade intervention through the distal radial artery, especially with small-diameter veins. Angioplasty balloons ranged from $2.5 \mathrm{~mm}$ (2$\mathrm{cm}$ long) to $4 \mathrm{~mm}$ (2- to $4-\mathrm{cm}$ long) in size and were manually inflated under direct visualization and palpation to avoid barotrauma venous injury. The inflation pressure required to dilate the veins ranged from 4 to 6 atm.

The length of the angioplasty ranged from 4 to 12 $\mathrm{cm}$ as measured by balloon length. This required two to three overlapping balloon angioplasty dilatations per vein beginning in the mid- forearm and progressing toward the spatulated end of the vein. For the final angioplasty, the final $5 \mathrm{~mm}$ of the balloon traversed the spatulated end of the vein and protruded out of the heel of the endto-side fashioned anastomosis to ensure complete dilatation (Figures 1: A,B).

Before the anastomosis was performed, veins were irrigated with a heparin flushing needle to hydrostatically dilate the transition between the angioplastied and nonangioplastied vein. The endto-side anastomosis of the AVF was completed after the angioplasty. Two hemostatic clips were placed on the tails of the anastomotic sutures to mark the location of the anastomosis for fluoroscopic identification during future planned BAM. PBA was performed over a length of vein spanning 4 to 12 $\mathrm{cm}$ above the spatulated end of the vein. After completion of the anastomosis, the transition zone between the angioplastied and non-angioplastied segment of the vein was examined to look for spasm. Any spasm in this region was treated by mechanical compression of the vein cephalad to the area of concern. Additionally, we found that placement of a tourniquet was helpful to facilitate early dilation of the vein after completion of the anastomosis. A sterile Penrose drain was used as a tourniquet in the operating room.

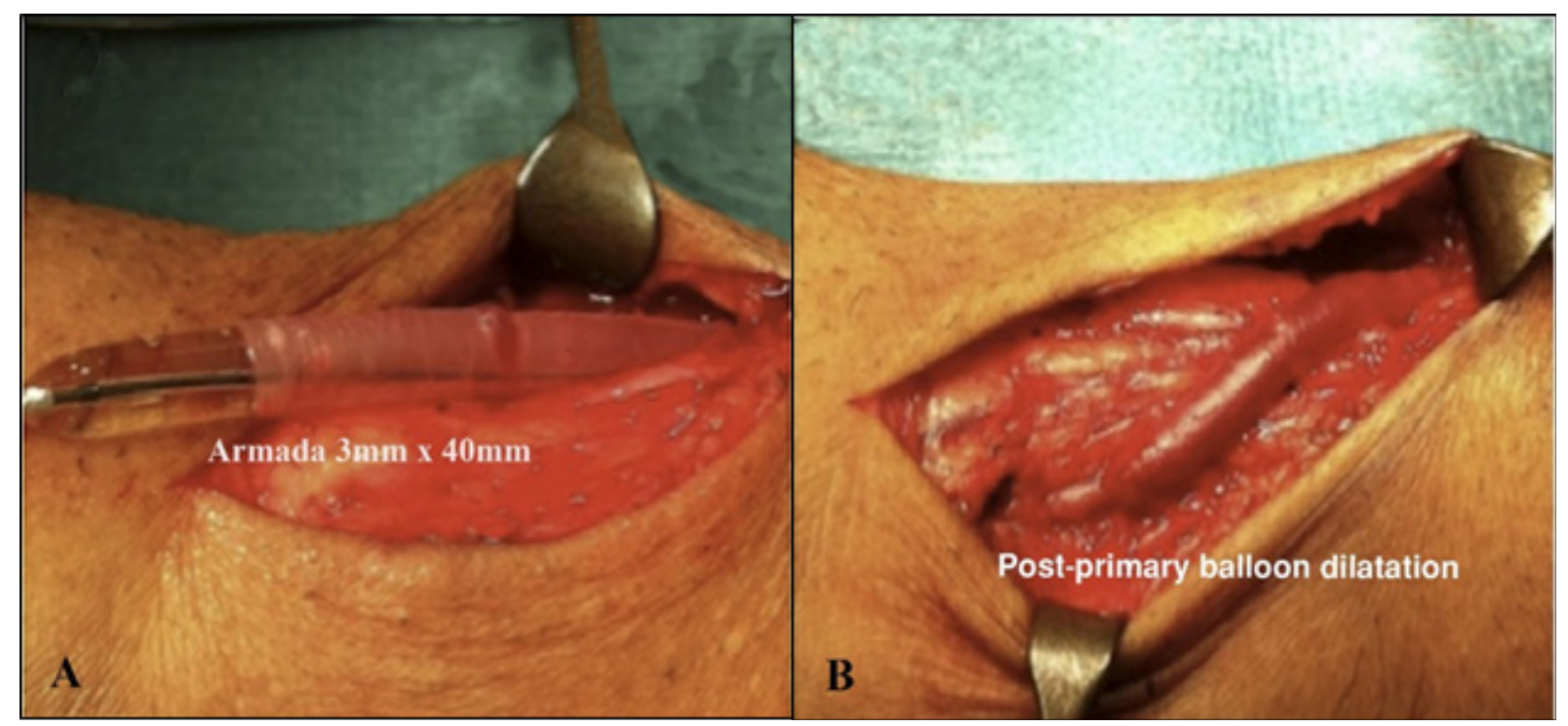

Fig 1: A, Primary balloon dilatation of the spatulated segment of the anastomosed cephalic vein. B, Post-anastomotic view of the dilated \& working radiocephalic fistula (AVF). 
The BAM technique included three major techniques: staged sequential dilation; controlled vein injury; and flow re-routing (Figures 1,2). The staged sequential dilation was performed by cannulating the AVF using the micro-puncture technique followed by insertion of a $4 \mathrm{~F}$ to $7 \mathrm{~F}$ sheath.

A retrograde radial artery approach was used for the initial BAM in most of the forearm AVFs. A 4F sheath and a $0.018^{\prime \prime}$ profile system were used to angioplasty the inflow or peri-anastomotic segment to $4 \mathrm{~mm}$ and the usable segment to 4 to $6 \mathrm{~mm}$ with the initial BAM performed 2 weeks after the creation of the AVF. Subsequent dilations were performed with 0.035 " profile systems using antegrade or retrograde sheaths placed into the proximal or distal AVF, respectively. Larger profile sheaths were used to accommodate the appropriate-sized balloons for treatment of any associated central venous stenosis.

The maturation of the usable segment of the AVF involved incremental balloon angioplasties of no more than 2 to $4 \mathrm{~mm}$ per session. In addition, the peri-anastomotic inflow region was angioplastied using a 4-mm balloon in almost all patients at the initial BAM. Patients with an initial inflow occlusion or veins $<2 \mathrm{~mm}$ in diameter were angioplastied with a 3-mm balloon at the initial BAM and then to $4 \mathrm{~mm}$ at the subsequent BAM. All outflow stenoses were treated appropriately with angioplasty. Recoil in the outflow veins was treated with high-pressure balloons (>30 atm) and stenting was reserved for resistant lesions. A post-procedure fistulagram was then obtained of the entire AVF, including the treated segments. Additional dilations were performed 2 to 4 weeks later, as needed, with the goal of increasing the usable segment diameter to 8 to $10 \mathrm{~mm}$ for forearm AVFs and 10 to $16 \mathrm{~mm}$ for upper arm AVFs.

End points for completion included the goal vein diameter and an AVF that functioned for dialysis 30 days without complications. The usable segment is defined as the maximal usable length of the AVF available for the "draw" and "return" needle sites (i.e., segment available for cannulation).

The concept of "usable segment maturation" is the most important component leading to the expedited maturation of an AVF. The concept of usable segment maturation is pertaining to AVF maturation using percutaneous techniques, as it is the underlying principle of sequential BAM. Flow re-routing was performed in patients with forearm AVFs with multiple outflow channels. Flow re-routing involved BAM of the intended primary venous outflow channel. The ultimate goal of the flow re-routing was a single, large-diameter outflow tract.

Collaterals within the usable segment frequently regress during BAM due to over-dilation of the involved area and, therefore, they rarely required intervention. Proximal collaterals are frequently ligated at the time of AVF creation, especially if they are large or located within $6 \mathrm{~cm}$ of the anastomosis.

However, these more proximal or "inflow" collaterals are not sacrificed with small-diameter veins, especially those requiring a PBA with a balloon $<3 \mathrm{~mm}$ in diameter. Of the 136 PBA procedures, 119 patients underwent subsequent BAMs on weeks 2, 4, and 6 to aid in maturation of the usable segment of the AVF. Initial BAMs were performed under radiologic guidance with a retrograde radial arterial approach using a 0.018inch platform at week 2 and an antegrade proximal fistula approach on a 0.035 - inch platform at weeks 4 and 6 . Sequential balloon dilatation of the entire fistula was performed 2 to $3 \mathrm{~mm}$ larger than the measured vein diameter at each BAM session. Ballooning was performed using an inflation device with an average pressure of $12 \mathrm{~atm}$. The venous side of the anastomosis, which had undergone PBA, was routinely angioplastied to 3 to $4 \mathrm{~mm}$ at the initial BAM. The length of the AVF used for access (venipuncture) was sequentially dilated to an average diameter of $8 \mathrm{~mm}$ for forearm AVFs and to 10 to $16 \mathrm{~mm}$ for upper arm AVFs. Sites of extravasation were limited and controlled during the angioplasty. Success was defined as the ability to use the fistula for hemodialysis without revision for 90 days. All fistulas were used $\leq 2$ weeks of the final BAM. All patients underwent routine clinical surveillance and radiologic evaluation, when indicated, in an attempt to prevent late failure of the AVF. Angiography was performed based on evidence of venous hypertension, decreased flow rates at hemodialysis ( $<250 \mathrm{~mL} / \mathrm{min})$, diminished or absent thrill at the fistula, and prophylactically at 3- to 6-month intervals as determined by clinical indications. 
Table 1: Diameters of the used veins

\begin{tabular}{cc}
\hline Number of performed procedures & Diameter of vein (mm) \\
\hline 44 & 3 \\
17 & 2.5 \\
57 & 2 \\
12 & 1.5 \\
6 & 1 \\
\hline
\end{tabular}

Statistical analysis was performed with the SPSS Statistics (Version 22, IBM Corporation, New York, USA) and plots were produced with the Graph-Pad
Prism statistical software package (Version 6.01; GraphPad Software, La Jolla, CA, USA).
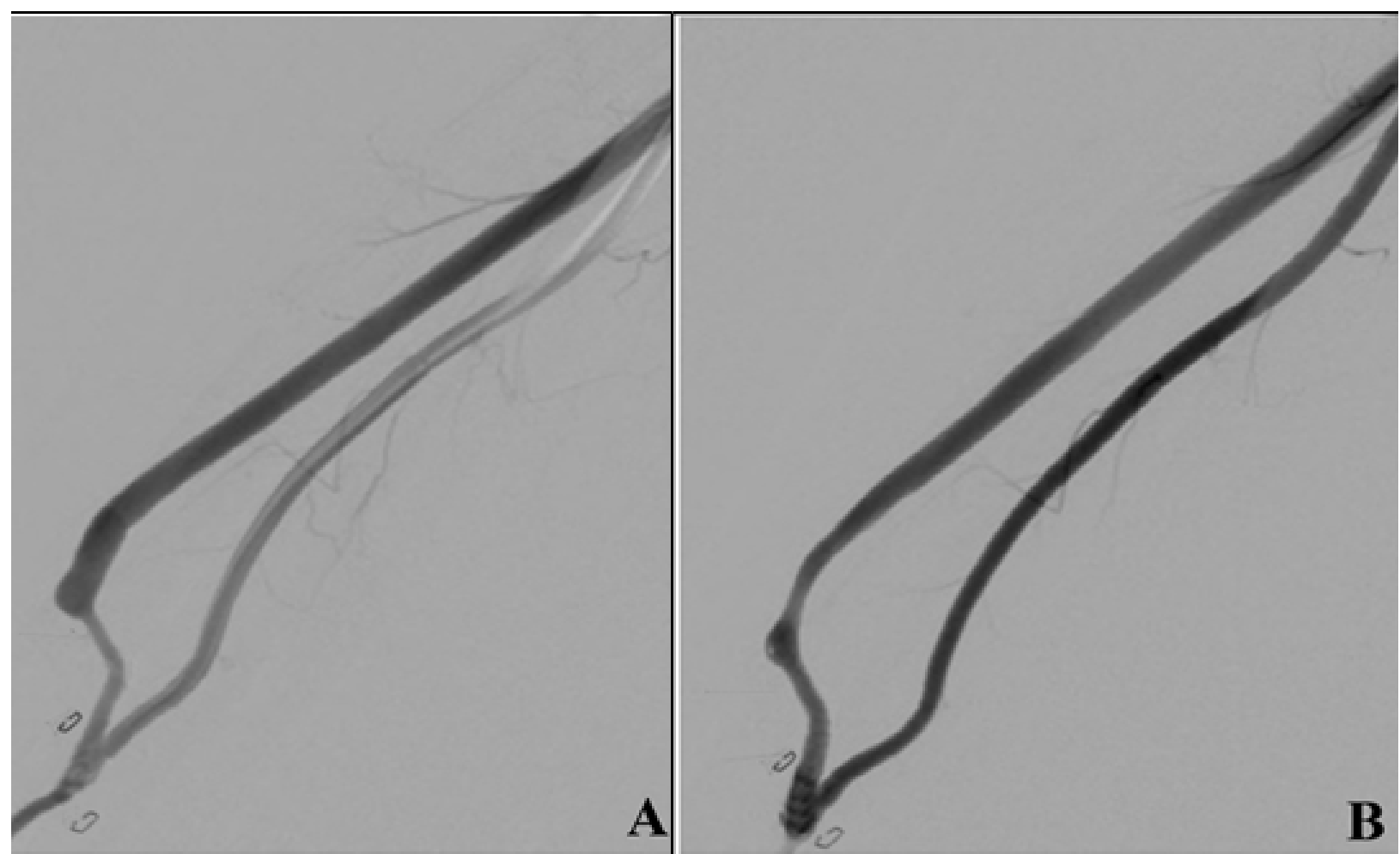

Fig 2: Successful fistula maturation after first BAM in a 61-year-old female with a delayed-maturing autogenous radiocephalic AVF; A, Initial angiography shows a focal severe stenosis (bracket) in the juxtaanastomotic vein of AVF; B, Angiography performed after the salvage BAM shows overall improvement of the stenosis, with subtle residual lesions (bracket) in the juxta-anastomotic vein of the AVF. 


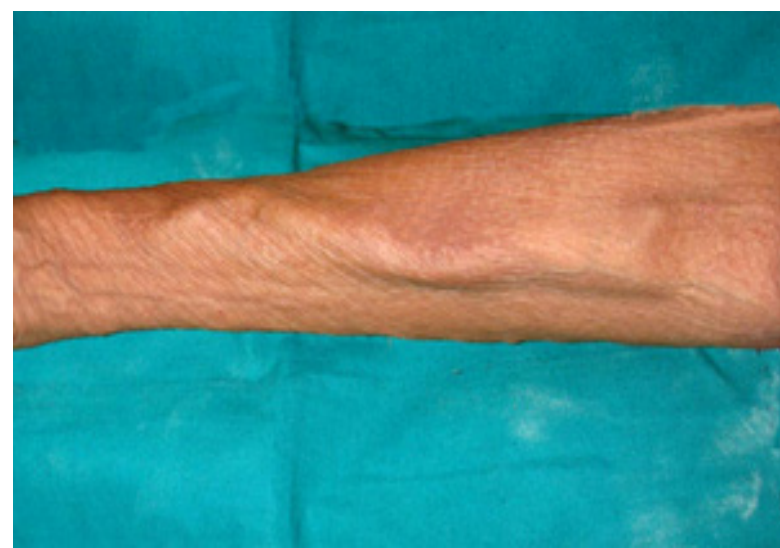

Fig 3 (A): Right radio-cephalic AVF 6 weeks after BAM with well-dilated forearm cephalic vein with readiness for start of hemodialysis.
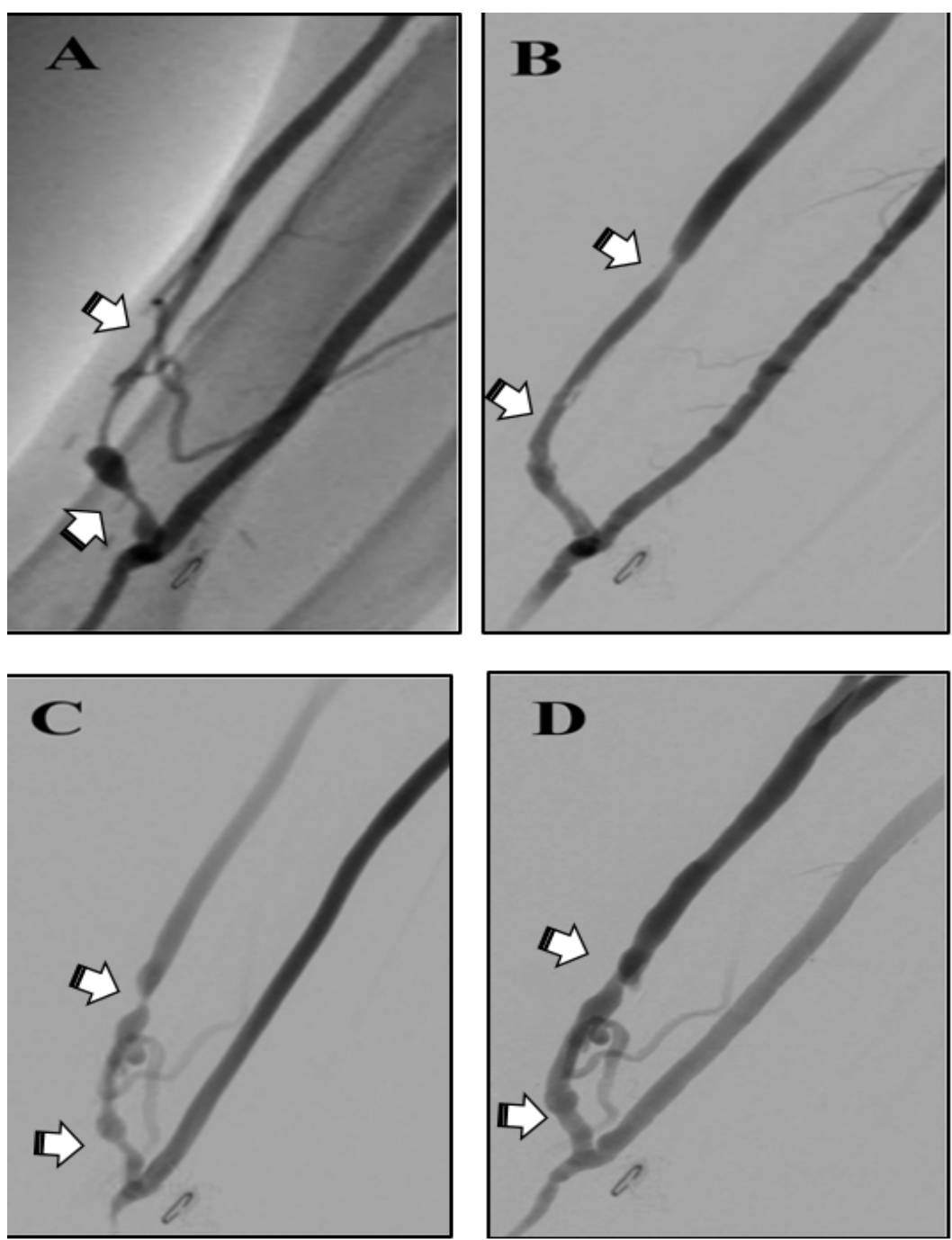

Fig 4: Sequental BAM of a delayed maturing radiocephalic AVF; A, Initial angiography showed a focal severe stenosis (arrow) in the juxta-anastomotic vein of the AVF; B, Angiography performed after the first BAM show improvement of the stenosis with subtle residual lesions (arrow) in the juxta anastomotic vein the AVF. C, A second BAM four weak after the first salvage session releaved the recurrence of sever multifocal stenosis (arraw) in the juxta-anastomotic vein of the AVF and weak accessory veins. D, Angiography after the second salvage BAM showed significant improvement of the stenosis with subtle residual lesions (arraw) in the juxta anastomotic vein of the AVF. 


\section{Results}

In our case series study 121 patients (69 men, 52 women) underwent PBAs. Mean age for the enrolled patients was 67.3 years (range, 49-88 years). Of these, 35 patients were not currently hemodialysis-dependent \& our constructed AVF was the first hemodialysis access for them, and 86 patients were currently on hemodialysis and had at least one previous AVF/AVG (were multiple in 11 patients). Previous ipsilateral access was constructed in 50 patients. One hundred and thirty-six procedures were performed, 85 BresciaCimino fistula type, 33 mid-forearm radio-cephalic, 16 antecubital/cephalic vein to brachial artery, and 2 accessory (posterior accessory vein to radial artery) fistula were created. The major component of this PBA patient population comprised native, sclerotic, small-diameter tortuous vein segments. In 4 patients with totally occluded sclerotic veins, guidewire cannulation, recanalization and PBA were performed, resulting in functional fistulas. Of the 136 PBA procedures, 119 (87.5\%) remained patent and subsequently underwent BAM with a resulting functional AVF. These consisted of 106 of the original AVFs and 13 new AVFs created at other sites. Occlusions occurred in 28 of the 119 functioning fistulas (23.5\%), and 15 were salvaged using BAM techniques. The mean balloon diameter for all dilatations was $3.5 \mathrm{~mm}$. so of the initial 121
AVFs placed, 106 (87.6\%) were made functional. 30 failed fistulae due to occlusion, fifteen were salvaged using recanalization techniques and sequential BAM. Of the remaining fifteen patients, 11 were un-salvaged and received AVFs at another site using PBA and BAM techniques and 4 required graft placement. One AVF had to be ligated secondary to steal syndrome with insertion of chronic hemodialysis catheter as an alternative access. Overall, 106 of 121 patients (87.6\%) obtained a working AVF at the initial site, and 116 (95.9\%) received working AVFs. All fistulas were functioning at 90 days after the final BAM. The duration of use of each fistula for hemodialysis is shown on the Kaplan-Meier curve and table analysis (Figure 5) \& time vs standard error (SE) (Table 2). Graft placement was necessary in only 4 of the 121 patients (3.3\%) who had intraoperative PBA, followed by BAM. Fistulograms during maturation demonstrated healing signs such as lack of contrast extravasation and smooth wall lining in all veins at both the PBA site and the entire length of the usable segments of fistulas subjected to sequential balloon maturations. PBA sites that were salvaged after occlusion (15 cases) and ballooned to a 4-mm diameter at the initial maturation-maintained patency and demonstrated angiographic features of healing.

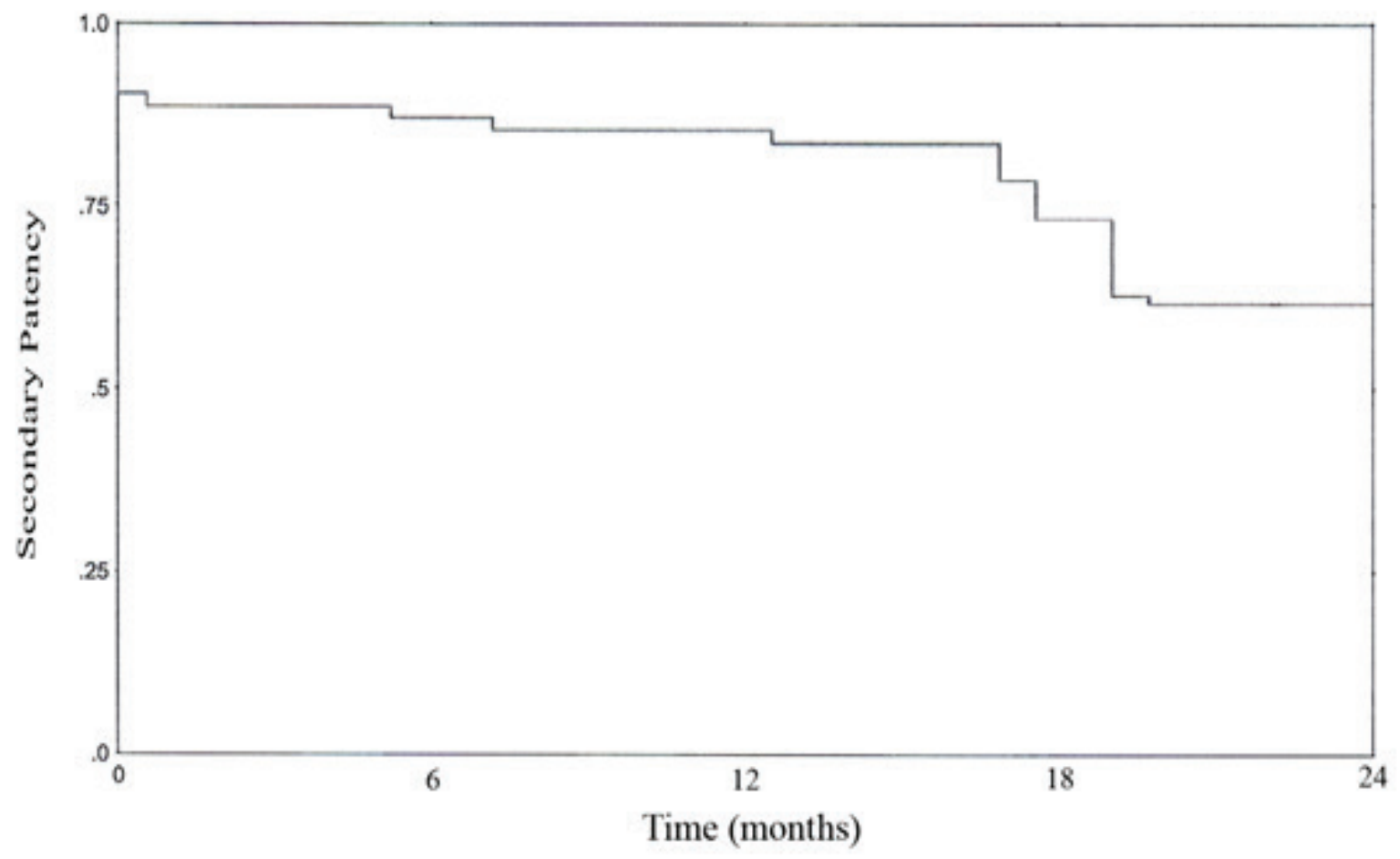

Fig 5: Kaplan-Meier curve shows cumulative secondary patency vs time in months. 
Table 2: Time vs standard error (SE)

\begin{tabular}{ccc}
\hline Time (months) & Remain at Risk & S.E \\
\hline 3 & 121 & 4.02 \\
6 & 121 & 4.02 \\
9 & 118 & 4.21 \\
12 & 116 & 4.43 \\
15 & 116 & 4.43 \\
18 & 114 & 4.42 \\
21 & 79 & 4.71 \\
24 & 46 & 4.70 \\
\hline
\end{tabular}

\section{Discussion}

Although the concept of increasing the number of AVFs is relatively well-accepted, advances in techniques and approaches to achieve the recommended guidelines are lacking. The trend of utilizing less than optimal vein segments has resulted in an increased number of inadequate AVFs. ${ }^{20,21}$ Thrombosis, low flow, difficult cannulation, rupture, poor maturation, longer maturation times, and shorter patency rates of suboptimal AVFs have resulted in an increased need for percutaneous and surgical revisions. ${ }^{22}$ Although initial numbers can be increased, sustained access for dialysis must be achieved for the AVF to be considered successful. The current emphasis on increasing the prevalence of AVFs must be addressed with a wider armamentarium of treatment options for failing or non-maturing AVFs. ${ }^{23,24}$ The use of suboptimal veins for the construction of the AVF has also been associated with increased maturation times..$^{10}$ Notably, the KDOQI guidelines recommend a maturation period of 2 to 3 months to avoid injury to an immature autogenous access. ${ }^{1}$ Literature researches have demonstrated that the use of small-caliber veins for vascular access is directly related to the number of revisions and complications. . $^{25,26}$ Using smaller veins for AVFs has resulted in an increased number of fistula revisions and an increased number of non-matured fistulas. ${ }^{27}$ Allon and Robbin ${ }^{28}$ reported veins $<2.5$ $\mathrm{mm}$ in diameter to have poor outcomes, and recommendations for not using smaller-diameter veins have been established. In our study, we are extending the statement to the $3-\mathrm{mm}$ veins in our enrolled patient group since we measured them in a wall-to-wall fashion. In our study with the conjoint two techniques, PBA \& subsequent BAM improve the results with suboptimal small-caliber veins $>3 \mathrm{~mm}$ in diameter. Previous techniques which used hydrostatic dilatation in addition to the administration of local vasodilators have not been successful with such extremely smallcaliber veins. ${ }^{29}$ It is commonly encounter difficulty in placing pediatric feeding tubes ( $5 \mathrm{~F}$ to $8 \mathrm{~F}$ ) for hydrostatic dilatation in veins $>2 \mathrm{~mm}$. In the same context, cannulation and advancement of a Garret dilator is often challenging, repeatedly resulting in vein perforation. Increased shear trauma and counter traction tears can also occur when trying to mechanically dilate unforgiving vein segments. Hydrostatic dilatation demonstrates less effective radial force than primary angioplasty balloon dilatation and may not generate sufficient pressure due to the presence of collateral veins. Guidewire access to the venous conduit when PBA is used allows for easy balloon access, and sufficient force is generated through angioplasty to dilate small-diameter sclerotic vein segments. With PBA, the veins (4 to $6 \mathrm{~cm}$ from the anastomosis) then undergo intraoperative angioplasty with 2.5to 4-mm-diameter balloons before the end-toside anastomosis is performed. These veins are clearly injured, but balloon dilatation facilitates performing the anastomosis and subsequent maturation. Guidewire cannulation during BAM is also facilitated. Guidewire recanalization was feasible even in the occluded segments (15 cases in this study) due to the larger-diameter vein at the original PBA. These PBA segments angioplastied to a diameter of 3 to $4 \mathrm{~mm}$ provide an adequate inflow to the AVF. For forearm AVFs, the inflow of the fistula (which corresponds to the PBA segment) is not made larger than $4 \mathrm{~mm}$ and transitions to an 8- to $10-\mathrm{mm}$-diameter fistula in the area used for cannulation. The inflow for upper arm AVFs is also kept at $4 \mathrm{~mm}$ while transitioning to a 12 - to $16-\mathrm{mm}$ area for cannulation. We believe this morphology reduces the incidence and/or severity of steal. Only two patients with severe small vessel disease in the palmar arch and digital vessels required ligation of the AVF secondary to severe steal. The PBA allows enough inflow to maintain patency of veins. BAM to large-diameter AVFs has reduced the need for superficialization of these fistulas and has avoided the use of basilic vein transpositions in our experience. Arterialization of PBA and BAM segments heal mostly by fibrosis, and we postulate that these AVFs eventually become large-diameter 
fibrous segments. These fibrous AVFs have not demonstrated a propensity for aneurysmal degeneration. Healing of these segments is further supported by the appearance of these fistulas during the sequential fistulograms obtained during BAMs. Evidence of mild extravasation is shown to heal with a smooth lining on subsequent images. We have found that 2-week intervals provide an optimal time for healing between angioplasties, and no vein ruptures occurred during any of the PBA and BAM procedures. The process of focal angioplasty injury to the venous endothelium and subsequent healing process seen after PBA and $B A M$ helps the venous wall reorganize into a fibrous conduit. We postulate that the largerdiameter conduits contribute more to improved patency than the overall characteristics of the fistula wall, which are yet to be determined.

\section{Conclusion}

The combination between PBA and BAM is an overall approach that facilitates AVF maturation, improves function, prolongs patency, and facilitates use of small-caliber veins as an autogenous vascular access. The shorter maturation times have reduced the overall need for indwelling catheters with their associated risks. The resulting large-diameter AVFs are easy to cannulate and are associated with improved flow and patency rates. Successful AVFs have been created using veins that would have otherwise been deemed unusable. Adoption of this conjoint technique should help meet the stringent demands for autogenous access placement defined by KDOQI and Fistula First Breakthrough Initiative (FFBI) and, thereby, improve the duration and quality of life for the hemodialysis patients.

\section{References}

1. National Kidney Foundation: NKF-K/DOQI Clinical Practice Guidelines for Vascular Access: Update 2000. Am J Kidney Dis. 2001; 37: S137-S181.

2. Powell S, Chan T: Endovascular techniques for cannulation difficulties in dialysis access. $\boldsymbol{J}$ Vasc Access. 2014; 15: S96-100.

3. DerDerian $T$, Hingorani $A$, Ascher $E$, Marks $\mathrm{N}$, Jimenez R, Aboian $\mathrm{E}$, et al: To BAM or not to BAM?: A closer look at balloon assisted maturation. Ann Vasc Surg. 2013; 27: 104-9.

4. United States Renal Data System [Internet]: USRDS. [cited 2016 Jan 22]. Available at: http://www.usrds.org/2015/view/default.aspx.

5. Sidawy AN, Spergel LM, Besarab A, et al: The Society for Vascular Surgery: Clinical practice guidelines for the surgical placement and maintenance of arteriovenous hemodialysis access. J Vasc Surg. 2008; 48: S2-25.

6. Howard $A D$, Howard RS, Goldstein $S L$, et al. Fistula First Breakthrough Initiative (FFBI): Lessons about arteriovenous fistula prevalence goals. Am J Kidney Dis. 2013; 61: 523-5.

7. Betjes MGH, Agteren MV: Prevention of dialysis catheter-related sepsis with a citratetaurolidine-containing lock solution. Nephrol Dial Transplant. 2004; 19: 1546-51.

8. Ravani $\mathrm{P}$, Palmer SC, Oliver $\mathrm{MJ}$, et al: Associations between hemodialysis access type and clinical outcomes: A systematic review. J Am Society Nephrol. 2013; 24: 46573.

9. Asher E, Glade P, Hingorani A, et al: Changes in the practice of angio-access surgery: Impact of dialysis outcomes and quality initiative rec-ommendations. J Vasc Surg. 2000;31:8490.

10. Patel ST, Hughes J, Mills JL: Failure of arteriovenousfistula maturation: An unintended consequence of exceeding Dialysis Outcome Quality Initiative Guidelines for hemodialysis access. J Vasc Surg. 2003;38:439-445.

11. Allon M, Robbin ML: Increasing arteriovenous fistulas in hemodialysis patients: Problems and solutions. Kidney Int. 2002;62:1109-24.

12. Huijbregts $\mathrm{HJ}$, Bots $\mathrm{ML}$, Wittens $\mathrm{CH}$, Schrama YC, Moll FL, Blank-estijn PJ, et al: Hemodialysis arteriovenous fistula patency revisited: Results of a prospective, multicenter initiative. Clin $\boldsymbol{J}$ Am Soc Nephrol. 2008;3:714-9.

13. Mclafferty RB: Techniques to enhance arteriovenous fistula maturation. Perspect Vasc Surg Endovasc Ther. 2009;21:41-5.

14. Miller G, Goel N, Khariton A, et al: Aggressive approach to salvage non-maturing arteriovenous fistulae: a retrospective study with follow-up. J Vasc Access. 2009;10:183-91.

15. Miller G, Friedman A: Balloon-assisted maturation of arterio-venous fistula. Endovasc Today. 2010; 46-54.

16. Garcia LPDM, Davila-Santini LR, Feng Q, et al: Primary balloon angioplasty plus balloon angioplasty maturation to upgrade smallcaliber veins $(<3 \mathrm{~mm})$ for arteriovenous fistulas. J Vasc Surg. 2010; 52: 139-44.

17. Derderian $T$, Hingorani $A$, Ascher $E$, et al: To 
BAM or not to BAM? A closer look at balloonassisted maturation. Ann Vasc Surg. 2013; 27: 104-9.

18. Miller GA, Schur I, Song M, Rodino W, Tarantini $F$, Jatwani $M$, et al: Balloon angioplasty maturation of arteriovenous fistulae: A new technique to facilitate placement and utilization of primary arteriovenous fistulae. Vascular. 2005; 13: S80-1.

19. Robbin ML, Gallichio $M H$, Deierhoi $M H$, Young $\mathrm{CJ}$, Weber TM, Allon M: US vascular mapping before hemodialysis access placement. Radiology. 2000; 217: 83-8.

20. Biuckians A, Scott EC, Meier GH, et al: The natural history of autolo-gous fistulas at firsttime dialysis access in the KDOQI era. $\boldsymbol{J}$ Vasc Surg. 2008; 47: 415-421.

21. Wolford HY, Hsu J, Illig KA, et al: Outcomes after autogenous brachial-basilic upper arm transpositions in the post-National Kidney Foundation Dialysis Outcomes Quality Initiative era. J Vasc Surg. 2005; 42: 951-956.

22. Bakken AM, Protack CD, Saad WE, et al: Longterm outcomes of pri-mary angioplasty and primary stenting of central venous stenosis in hemodialysis patients. J Vasc Surg. 2007; 45: 776-783.

23. Surowiec SM, FegleyAJ, Tanski WJ, et al:
Endovascular management of central venous stenoses in the hemodialysis patient: Results of percutaneous therapy. Vasc Endovascular Surg. 2004; 38: 349-354.

24. Greenberg JL, Suliman A, Angle N: Endovascular dialysis interventions in the era of DOQI. Ann Vasc Surg. 2008; 22: 657-662.

25. Berman SS, Gentile AT: Impact of secondary procedures in autogenous arteriovenous fistula maturation and maintenance. $\boldsymbol{J}$ Vasc Surg. 2001; 34: 866-71.

26. 26. Robbin ML, Chamberlain NE, Lockhart ME, Gallichio $\mathrm{MH}$, Young $\mathrm{CJ}$, Deierhoi $\mathrm{MH}$, et al. Hemodialysis arteriovenous fistula maturity: US evaluation. Radiology 2003;225:59-64.

27. Ascher $E$, Gade $P$, Hingorani A, Mazzariol $F$, Gunduz Y, Fodera M, et al: Changes in the practice of angioaccess surgery: Impact of dialysis outcome and quality initiative recommendations. J Vasc Surg. 2000; 31: 8490.

28. Allon $M$, Robbin ML: Increasing arteriovenous fistulas in hemodialysis patients: Problems and solutions. Kidney Int. 2002; 62: 1109-24.

29. Ramos JR, Berger K, Mansfield PB, Sauvage LR: Histologic fate and endothelial changes of distended and nondistended vein grafts. Ann Vasc Surg. 1976; 183: 205-28. 